\title{
Clinical pearls: juvenile diabetics mellitus
}

\section{Introduction}

Type 1 diabetes mellitus occur in children $\&$ is insulin dependent. It is uncommon but serious disease as compared with type 2 diabetes. Type 1 diabetes mellitus has 2 peak occurance: one at 5years \& second at 5 to 10 years.

The exact cause is not known. It may occur because of autoimmune destruction of $\beta$ cells of pancreas. The disease may occur following a viral infection (mumps, coxsackie, cytomegalo), early introduction of cow millk protein may be a important factor in the subsequent development of diabetes in genetically susceptible children (HLA DR3 \& HLA DR4). Environmental toxins and reactive oxygen free radicals may cause damage to the islets cells. Ther is evidence to suggest thatvitamin D supplementation in early childhood may offer protection against the development of type 1 diabetes mellitus. There is increasing incidence of type 2 diabetes in adolescent children with obesity (metabolic syndrome X)and girls with polycystic disease of overies.

\section{Clinical expressions}

Disease may occur at any age but it is more common in school going children. There is marked elevation of blood glucose level which leads to excessive urination, marked thirst and increased appetite. Dispite excessive intake there is weight loss. At times, the disease may manifest as an emergency diabetes ketoacidosis or diabetic coma. There may be sudden episode of vomiting, abdominal pain, fever, dehydration, drowsiness or coma. The proportion of recently diagnosed children may go in to spontaneous remission and it may last from few months to 2years (honeymoon phase). This is followed by relapse with progressive increase in insulin requirement, which may reach to a level. 8 to 1 unit $/ \mathrm{kg} /$ day. The classical triad of polyuria, polydypsia and polyphagia with failure to thrive is diagnostic.

\section{Laboratory parameters}

The diagnosis of DKA confirmed by presence of hyperglycemia $(>250 \mathrm{mg} / \mathrm{dl})$, ketonuria. Ketonemia with blood $\mathrm{Ph}<7.3$ and base excess more than -20 . There is hypertriglyceridemia, hyperkalemia and hyperosmolality $(>350 \mathrm{mOsm} / \mathrm{Land}$ increased anion gap $(>12 \mathrm{mEq} / \mathrm{L})$. Urine analysis shows presence of sugar and ketone bodies. Moderate leucocytosis may occur because of stress and severe leukocytosis $\left(>25000 / \mathrm{mm}^{3}\right)$ suggestive of associated infection. X-ray chest exclude pneumonia and CSF examination exclude memingoencephalitis.

\section{Monitoring during treatment of DKA}

a. Vital signs, ECG \& S.electrolytes.

b. Fluid intake \& out put chart

c. Capillary blood glucose with glucometer every hour.

d. Blood glucose, electrolytes, blood urea, blood $\mathrm{Ph}$ and osmolality every 2-4hours.

e. Clinical status, vitalsigns, level of consciousness, status of hydration and evidence of cerebral oedema (rise of blood pressure, bradicardia, $6^{\text {th }}$ nerve palsy, papillary sizeand respose to light.

\author{
Volume 6 Issue I - 2018 \\ Bharat Parmar \\ BJ Medical College Civil Hospital, India
}

Correspondence: Bharat Parmar, Professor of Head of Unit (Pediatrics), BJ Medical College, Civil Hospital Ahmedabad, Gujarat, India,Tel +9198256|3362, Email bjpdr@hotmail.com

Received: March 15, 2017 | Published: January 30, 2018

\section{Management}

Fluid therapy: Insulin therapy: Exercise and physical activity: Diet \& Nutrition

Fluid therapy: Initial management of with normal saline $10 \mathrm{ml} / \mathrm{kg}$ over 1hour. Maintainance fluid along with deficit is given slowly over a period of 24 to 36 hours. Agressive fluid therapy should be avoided because of risk of development of cerebral oedema. When blood glucose fall below $300 \mathrm{mg} / \mathrm{Dl}$ or less. Potassium is added to the infusate $(40 \mathrm{mgL} / \mathrm{L})$ once child passed urine. There is no need to correct acidosis by administration of sodium bicarbonate unless $\mathrm{Ph}$ is $<7.0$ One third of the calculated amount of sodium bicabonateshould be administered over 1 hour. Antibiotics are given if superadded infection.

Insulin therapy: Soluble insulin is given as a bolus dose of 0.1unit/ $\mathrm{kg}$ intravenously followed by continuous intravenous infusion at a rate of 0.1 unit $/ 1 / \mathrm{hr}$. The currently available insulins are produced by recombinant deoxyribonucleic acid technology. The usual insulin dose is 1 to 1.5 units $/ \mathrm{kg} /$ day and combination of intermediate acting (Lente) and short acting (regular) insulin given 20 to 30minutes before breakfast and dinner. Approximately two third of the dose is given as Lente insulin and one third dose as regular insulin (spilt-mix insulin). Insulin pen injectors are available for ease of administration. Injection are given subcutaneously and rotated between anterior abdominal wall and anterolateral parts of thigh and arms. The most accurate method of achieving glycemic control IV by use of an insulin pump. It utilize insulin delivery device to accurate deliver a base line contuneous infusion of insulin coupled with periodic bolus related to food intake and activiry level. Nasal spray of insulin is undergoing trials which are likely to simlyfy the treatment of diabetes.

Exercise and physical activity: Regular exercise and physical activity increases glucose utilization and enhances sensitivity of muscles to insulin. It improves metabolic control. The useful exercises include vigorous walking, jogging, and cycling, swimming and aerobic exercise.

Diet and nutrition: A diet plan with distribution of calories into three major meals and three mid-meal snacks to prevent hypoglycemia. $60 \%$ calories from carbohydrates, $15 \%$ from proteins and 30 to $35 \%$ from fats. Carbohydrates should be complex (instead of refined sugars) with low glycemic index and plenty of fibers (20 to $25 \mathrm{gm} /$ day). The naturally occurring sugar in fruits is tolerated better. Protein intake is lowered to $8 \%$ calories in children with microalbuminuria and diabetic neuropathy. Fat sources include one third saturated fats, 
one third polysaturated fatty acids and one third monosaturated fatty acids. The diet plan can be fine tuned with life style, physical activity, eating habits, and insulin therapy and health status of child.

\section{Complications}

Acute complications include intercurrent infection. Dyslipedemia, hypoglycemia \& DKA. Growth failure, delayed sexual maturation and impaired intellctual development. Chronic complications i.e. diabetic retinopathy, neuropathy $\&$ nephropathy.

\section{Monitoring \& follow up}

Urine testing for gucose protein and ketones of first morning urine sample once a month. Blood glucose should be checked with a glucometer before each meal and fasting. The fasting level should be maintained between $80 \mathrm{mg}$ to $120 \mathrm{mg} / \mathrm{dl}$ and random glucose level between 80 to $140 \mathrm{mg} / \mathrm{dl}$.

Glycosylated hemoglobin should be checked every 3 months and maintained below $8 \%$. It is excellent marker of adequacy of glucose controlover period of 6 to 8 weeks. Serum lipids cholesterol high density lipoprotein and very low density lipoprotein fraction and triglycerides should be checked once a year. Thyroid function TSH \& T4 should be checked once a year. Funds examination once a year.

\section{Clinical Analysis: Case series of type I diabetes}

Juvenile diabetes mellitus Type 1 case series: There were 3 new cases of type 1 diabetes and 2 known case of diabetes admitted in my paediatric ward in last 6 months. 28 months male child new case of diabetes admitted with diabetic ketoacidosis with upper respiratory tract infection. Glycosylated hemoglobin was $10.9 \% \mathrm{Hb} 10.0 \mathrm{gm} \%$ WBC 13800 Platlet count 4.9lacs.

4 year male child new case of diabetes admitted with diabetic ketoacidosis with UTI, anemia and PEM Glycosylated hemoglobin 9.9\% WBC 16000 cumm, Hb $10.5 \mathrm{gm} \%$ Urine 10 to 12 pus cells Urine culture positive for Candida RFT were abnormal. 6year male child new case of diabetes admitted with diabetetic ketoacidosis with UTI, Glycosylated hemoglobin was $8.9 \% \mathrm{Hb}$ $8.5 \mathrm{gm} \% \mathrm{WBC} 18500 \mathrm{cumm}$. Another 2 known case of diabetes admitted one was 5year female child diabetic ketoacidosis with nephrotic syndromre and Secon 5.5year old female child admitted with diabetic ketoacidosis with upper respiratory tract infection, anemia and undernutrition.

\section{Key messages}

a. Bed side blood ketone measurement is useful in diagnosis and monitoring of children with DKA.

b. Fluid therapy in DKA should always precede insulin infusion in DKA.

c. Ensure normal serum potassium before starting insulin infusion.

d. Restricted fluid therapy as per protocol is essential in management of DKA.

e. Rapid analogs of insulin can be used 1-2hourly if insulin infusion is not fesible.

f. Children on long acting insulin can continue the therapy during treatment of DKA.

g. Both new onset DKA \& recurrent DKA are preventable in children with diabetes.

\section{Acknowledgments}

None.

\section{Conflicts of interest}

None. 